\title{
Ethnologies
}

\section{Tourisme culturel en milieu insulaire}

\section{Le cas de l'Île aux Grues}

\section{Pascal Huot}

Volume 31, numéro 1, 2009

Passages

URI : https://id.erudit.org/iderudit/038503ar

DOI : https://doi.org/10.7202/038503ar

Aller au sommaire du numéro

\section{Éditeur(s)}

Association Canadienne d'Ethnologie et de Folklore

ISSN

1481-5974 (imprimé)

1708-0401 (numérique)

Découvrir la revue

Citer cet article

Huot, P. (2009). Tourisme culturel en milieu insulaire : le cas de l'Île aux Grues. Ethnologies, 31(1), 127-162. https://doi.org/10.7202/038503ar

\section{Résumé de l'article}

Facteur économique important, le tourisme joue un rôle déterminant pour l'île aux Grues. Cet article rend compte du potentiel touristique que l'île tire de sa situation géographique particulière. Peut-on y observer une manière de pratiquer un tourisme culturel qui soit du domaine propre à l'insularité ?

Comment les manifestations de la mi-carême et de la traversée en canot à glace entrent-elles en jeu dans la construction du tourisme culturel ? La promotion touristique fait-elle également place aux personnalités publiques comme le peintre Jean-Paul Riopelle, qui s'était établi dans le manoir de l'île ? L’auteur examine également l'impact du tourisme insulaire, tant sur les résidents de l'île que sur le touriste. S'appuyant sur des témoignages, il tente ainsi de mieux comprendre les implications que soulève la mise en marché d'un tourisme de masse dans un milieu restreint, sans pour autant sacrifier le quotidien de l'île pour plaire à tout prix.
Ce document est protégé par la loi sur le droit d'auteur. L'utilisation des services d’Érudit (y compris la reproduction) est assujettie à sa politique d'utilisation que vous pouvez consulter en ligne.

https://apropos.erudit.org/fr/usagers/politique-dutilisation/ 


\section{TOURISME CULTUREL EN MULIEU INSULAIRE Le cas de l'île aux Grues}

\section{Pascal Huot}

Université Laval

D'une région à l'autre, le tourisme diverge. Les moyens mis en œuvre afin de plaire aux touristes ainsi que les éléments qui entrent dans la promotion touristique changent, mais qu'est-ce qui préside à leur choix ? Le tourisme culturel constitue une manière de faire la promotion de sa culture dans le but d'attirer les touristes à la recherche de caractères distinctifs et locaux. Dans une communauté, qu'est-ce qui détermine le choix des faits et des manifestations qui ont façonné, modelé les paysages et l'histoire de ses habitants et qui sont ainsi mis à l'avant-plan pour se donner à voir à l'Autre ? Les choisit-on pour leur signification intrinsèque, pour les préserver du passage du temps? Selon Sara Le Menestrel, ces caractères propres à la communauté et choisis par celleci apparaissent également dans un besoin de pérennité : "Par contraste avec les autres formes de tourisme, le tourisme culturel est perçu par ceux qui en font la promotion comme un moyen de sauvegarder la culture, dont il tire ses ressources" (1999: 143). Au Québec, parmi la variété des milieux qui ont choisi la pratique du tourisme culturel, les îles habitées du Saint-Laurent ont-elles adopté un tourisme particulier? Peut-on y observer une manière de pratiquer un tourisme culturel qui soit propre au contexte insulaire? Et le touriste peut-il, en prenant part aux événements, en s'attardant aux particularités visuelles, mieux comprendre la population locale?

Le tourisme culturel façonne une image d'une région et de ses habitants, que celle-ci soit assumée ou non par la population locale. 
Cette représentation peut être un vecteur important dans la promotion touristique afin de toucher l'imaginaire des gens. Ce travestissement de l'image magnifiée est néanmoins un symbole à vocation fort utile quand vient le temps de faire la promotion d'une région, que cette image soit la réalité ou simplement des facettes d'une culture conservée pour des fins d'attraction touristique. Comme le souligne Karine Laviolette, " on comprend que le regard des touristes agit comme une force de différenciation culturelle, comme une forme de reconnaissance de la distinction. Ceci entraîne généralement une prise de conscience de l'altérité et pousse un groupe culturel à créer des lieux ou des événements investis d'un rôle identitaire" (2004: 260). Par ailleurs, on ne peut nier la force d'attraction opérée par la création de ces espaces ou événements à forte connotation identitaire. En effet, s'identifier à un milieu et l'investir de son histoire stigmatise l'image et permet sa reconnaissance hors de ses frontières. "Si on se croit perçu comme folklorique, c'est peut-être que parfois on se projette comme tel. La publicité touristique de Charlevoix n'est pas la seule à faire référence à une image préservée ou folklorique. Par exemple, celle du Saguenay et ses "bleuets" ou de Québec "vieille capitale française" et ses vieux murs n'en sont pas exemptes" (Gauthier 2003 : A9).

Dans la documentation ethnologique existante à ce sujet, les études portent principalement sur l'examen des phénomènes apparents, principalement ceux marqués dans le paysage ou visibles dans des manifestations de groupe (festivals, concerts, regroupements, associations...). Par exemple, dans son investigation de la ville de TroisPistoles, Laurier Turgeon a relevé des marques à même le paysage d'une forte identification au passage des Basques dans ce milieu. Il souligne notamment la rue des Basques, la Fromagerie des Basques, la commission scolaire des Basques, voire la Récupération des Basques, une entreprise spécialisée dans le recyclage. Le rapport qu'entretiennent les gens de Trois-Pistoles avec ce passé identitaire de la rencontre avec les pêcheurs basques se déploie ainsi devant le regard du badaud. Cette relation est encore renforcée par le Rendez-vous Basque', puisque dans ce cas, la

1. "La fête d'inauguration du centre muséographique du 13 juillet 1996, mieux connue sous le nom de Rendez-vous Basque, peut être interprétée comme une autre manifestation de la volonté d'incorporer dans le paysage patrimonial de la région une ethnicité manquante. La fête ne donne-t-elle pas l'occasion de mettre en scène de "vrais Basques" venus du Pays basque?" (Turgeon 2003 : 146) On invite des musiciens basques, des joueurs de pelote basque. Il y a même le directeur du Parc de l'Aventure Basque qui doit expliquer à la population locale ce qui est d'origine basque dans la fête. 
référence à la venue des Basques s'expose éloquemment. Les gens de Trois-Pistoles entretiennent aujourd'hui un fort lien avec le passage des Basques qui eut lieu du XV' au XVII siècle. De leur côté, les milieux insulaires peuvent-ils tabler sur un événement tout aussi marquant de leur histoire pour construire leur identité et développer une image culturelle? Ont-ils su développer et conserver une trace de leur mode de vie lié à leur situation géographique particulière? Bref, peut-on déceler dans l'offre touristique un caractère propre à l'insularité ?

Cet article propose d'examiner les éléments culturels mis en scène dans le tourisme culturel de l'île aux Grues². L'on peut présager que les insulaires se sont constitués une identité particulière, développant ainsi une façon propre d'entrer en relation avec les autres et le territoire. "Dans ce contexte, le citoyen (ou le groupe) est considéré comme un praticien de la culture dans son milieu. C'est à travers lui, ou à travers le groupe, que se développe le sentiment d'appartenance au milieu. L'enjeu n'est donc pas que culturel, il est aussi social, la culture de proximité étant un important facteur de cohésion sociale, d'ancrage au territoire " (Genest et Lapointe 2004 : 75). Embrasser l'insularité sous l'angle du tourisme culturel peut alors se révéler un moyen efficace d'entrer dans un univers où les gens mettent en valeur leur territoire et leur identité de manière à plaire à autrui, aux touristes en général, mais aussi à eux-mêmes, car il existe un fragile équilibre entre la satisfaction du touriste et la sauvegarde de sa spécificité. Comme le précise Sara Le Menestrel : "Les initiatives touristiques ne sont pas seulement destinées aux touristes, mais s'avèrent déterminantes dans la construction de l'identité du groupe " (1999: 155). Aussi, si certains éléments sont d'abord mis en place pour le tourisme et reviennent régulièrement dans le discours touristique, tel que la mi-carême, l'on peut se demander si d'autres éléments, plus discrets dans les outils promotionnels, rappellent l'histoire ancienne ou récente de ses habitants, tel que la traversée en canot à glace ou l'établissement du peintre Jean-Paul Riopelle dans le manoir de l'île. Peut-on déceler l'utilisation actuelle de ces manifestations insulaires dans la construction touristique de l'île?

2. Au cours de mes recherches, il m'a été possible de répertorier plusieurs orthographes de l'île aux Grues. Pour les besoins de cet article, j'ai préféré retenir celle-ci issue de la Commission de toponymie du Québec (2006: 260), la délaissant uniquement lorsque je cite des ouvrages contenant une orthographe divergente. Il est également à noter que lorsque j'utiliserai le diminutif "île ", celui-ci renvoie automatiquement à l'Île aux Grues. Pour une autre explication quant à cette toponymie, je réfère le lecteur à Jean O'Neil (1991: 50-51). 
Pour comprendre si l'insularité est un élément déterminant dans le discours promotionnel de l'île aux Grues, j'ai choisi d'examiner le point de vue des touristes, c'est-à-dire ceux à qui sont destinées les activités touristiques. Cette approche donne la parole à divers informateurs qui projettent d'aller sur l'île ou qui l'ont déjà visité. Johanne Ouellet, ornithologue amateur, originaire de Notre-Dame-du-Lac dans le BasSaint-Laurent, s'est établie à Montmagny depuis peu. Professeure de chant et étudiante en éducation spéciaiisée à Québec, elle projette d'effectuer un séjour sur l'île. Son témoignage permet donc d'évaluer les attentes et les préjugés d'une personne n'ayant jamais visité l'île. Clément Belley, retraité demeurant à Sept-îles, s'est rendu à l'île pour la chasse à l'oie une seule fois. Il apporte ainsi un point de vue de chasseur, la chasse étant considérée comme une forme de tourisme par les gens l'île. Bernadette Leblond, retraitée et habitant St-Lazare de Bellechasse, a fréquenté l'île en tant que touriste et elle donne son point de vue en tant que tel. Trois autres des informateurs rencontrés sont des intervenants directs auprès de touristes et ils livrent leurs impressions de l'expérience qu'on vécu ces derniers. Jean Gosselin, résident de Montmagny, est pilote pour Air Montmagny qui assure la liaison entre l'Île aux Grues et la terre ferme. Amélie Ringuet, originaire de Montmagny, est superviseure au bureau d'information touristique pour l'Office de tourisme de la Côte-du-Sud. Enfin, Gina Vézina est originaire de l'Île aux Grues et coordonnatrice du Musée Le Grenier de l'Isle. À l'analyse des discours s'est combinée une recherche documentaire en lien avec la promotion touristique de l'Île aux Grues (publicité, guide touristique...), ainsi qu'une observation in situ.

Dans cet article, je soutiens que l'insularité est déterminante dans la construction de l'identité et que le tourisme mis en place à l'île aux Grues véhicule cette identité distinctive. J'expose dans un premier temps les perceptions alimentées au sujet des îles pour se pencher plus spécifiquement sur le visage, la géographie de l'île aux Grues. J'observe également les attraits touristiques en place, notamment trois éléments liés à l'identité des gens de l'île aux Grues: la mi-carême, la traversée en canot à glace et le passage du peintre Jean-Paul Riopelle ${ }^{3}$. Enfin, l'analyse se concentre sur les répercussions d'un tourisme culturel

3. Le choix de ces trois éléments ne tend aucunement à l'exhaustivité. Ils ont été retenus pour les liens manifestes qu'ils entretiennent avec l'île aux Grues et, par conséquent, pour le caractère insulaire et distinctif qu'ils portent en eux. Cependant, d'autres thèmes pourraient être développés dans une étude subséquente, tel que l'histoire maritime de l'île. 
insulaire, tant chez les touristes que les perceptions qu'ils ont de leur passage sur ce territoire.

\section{L’imaginaire porté par les îles}

La fascination pour les milieux insulaires repose sur une image d'un lieu préservé, un endroit où la culture est restée inchangée, où le pittoresque côtoie l'image surfaite de l'immuabilité, faute de contact avec l'extérieur. Le mot île séduit ; c'est un nom commun qui désigne une entité territoriale, une identité insulaire et un mode de vie propre, "un pays dans le pays" (Pelletier 2003 : 94). Musée vivant, les îles sont-elles vouées à une conservation d'elles-mêmes à l'identique, une identité figée dans ces traits culturels? La culture cinématographique et télévisuelle, qui semble avoir saisi ce potentiel en mettant les spectateurs en relation avec le milieu insulaire, a-t-elle créé une image stigmatisée des îles? Ce faisant, les îles se transforment-elles en un produit, en une représentation stéréotypée ? Il ne faut pas remonter très loin dans le temps pour observer l'impact du cinéma sur l'industrie du tourisme culturel en milieu insulaire. Citons comme exemple le cinéma documentaire de Pierre Perrault (1963), qui a provoqué un tel engouement. Les gens partaient à l'Île aux Coudres à la recherche de cet isolement séculaire, mais aussi, ils allaient à la rencontre des protagonistes, comme en témoigne Pierre Perrault lui-même: "J'ai d'ailleurs pu constater que des tas de gens, à partir du film, se donnaient la peine d'aller voir [Marie] à l'Île aux Coudres .... Des autobus entiers s'arrêtaient devant sa porte" (Perrault 1996: 138). Pour le cinéaste, "l'île lui apparaissait être la source d'un riche patrimoine vivant, de traditions québécoises pratiquement disparues, telle que la fête de la Mi-Carême. En fixant sur la pellicule les récits de conteurs comme Alexis Tremblay, Perrault a indéniablement renforcé le mythe " (Dubé 2000 : 27). Plus récemment, l'île d'Harrington Harbour, sur la BasseCôte-Nord, a vu apparaître un nouveau marché pour la mise en valeur d'un "vrai faux village » par le biais du cinéma de fiction. Elle a ainsi pu saisir au passage une nouvelle clientèle de touristes qui a été séduite par le film La grande séduction (Pouliot 2004). "Les projecteurs qu'a braqués le film à succès sur le village ont eu pour résultat d'y amener de nouveaux contingents de visiteurs. Une maison ancienne vient d'être restaurée afin d'accueillir les touristes et de leur présenter l'histoire du village. Les deux seuls Bed \& Breakfast ne suffiront plus à ce rythme» (Simier 2005 : 29A). Aujourd'hui, l'île d'Harrington Harbour possède 
un atout à exploiter et elle le valorise, sans avoir nécessairement l'aval de toute la population, tel que le démontre un reportage du magazine télévisuel d'information Méchant contraste sur "Le sacro-sainttourisme ${ }^{4}$ (Caron 2005).

Certaines îles tirent profit de ce potentiel touristique valorisé par le septième art. Une telle publicité procure un important contingent de touristes, mais qu'en est-il lorsqu'une île doit vendre sa couleur spécifique sans l'artifice promotionnel grand public que fournit le cinéma? Comment rendre compte de soi, comment se présenter à l'Autre? Certes, un regard a été porté sur l'Île aux Grues ; bien des imaginaires ont investi les lieux, nourris par la littérature (Béchard 1902 ; O’Neil 1991) et rendus visibles par la photographie (Croteau 1995 ; Bouchard 2004), ainsi que par le cinéma ${ }^{5}$ (Garceau 1958 ; Lavoie 1963). Mais inévitablement, toutes ces pratiques se sont transformées. À sa manière, l'île est entrée dans la modernité. Il s'avère donc essentiel d'analyser les traits culturels de l'île aux Grues à la lumière de son contexte géographique et économique.

\section{Un contexte géographique et économique particulier}

"Une île est par définition un espace clos, continu et parfaitement limité. Sur cet espace se rassemble selon un processus historique une communauté ethnique la plupart du temps homogène et dont les membres acceptent de vivre et de se reproduire ensemble " (Defert 1988 : 2). Situé dans la région de la Côte-du-Sud, région touristique de Chaudière-Appalaches, à la hauteur de Montmagny, l'archipel de l'Île aux Grues ${ }^{6}$ compte un chapelet de vingt et une îles et îlots (figure 1), dont les plus connues sont la Grosse-île et l'île aux Grues. L'île aux Grues est aujourd'hui la seule habitée à l'année et la paroisse de Saint-

4. Ce magazine d'information aborde plusieurs sujets différents - six dans ce casci - face au thème de l'émission. Dans le cas du village d'Harrington Harbour, le reportage s'interroge, deux ans après la sortie du film La grande séduction, sur ce que devient la population face aux touristes de plus en plus présents.

5. Les documents qui ont été tournés à l'île n'ont pas eu de diffusion à grande échelle comme les deux exemples cités plus haut.

6. Cet archipel a déjà porté les noms d'archipel de Montmagny et d'archipel de la Côte-du-Sud ; cette deuxième appellation, selon Deschênes (1991: 71) et Vézina (1994: 16-17) serait plus appropriée. 
Antoine-de-l'Isle-aux-Grues compte à peine cent cinquante? âmes. L'île aux Grues est reliée à l'Île aux Oies, une île privée, par le chemin de la Batture (figure 2). Au bout de ce chemin, le visiteur est accueilli par une barrière solidement ancrée en vue de couper la voie aux visiteurs inopportuns (figure 3); le touriste ne peut y avoir accès. L'acquisition d'îles par des individus peut soulever des appréhensions:

Bien j'ai bien peur, moi, je le sais pas là, rendue à mon âge, je ne verrai pas ça, mais j'ai bien peur que ça va se vendre, s'il n'y a pas d'autres relèves. Le gouvernement va embarquer là-dedans ou un homme bien riche bien riche, et puis ça va être rien que pour eux autres, fait que y'aura plus de touristes là. Je ne le souhaite pas, mais j'ai bien peur (Bernadette Leblond).

Si l'Île aux Oies est privée, le chemin pour y accéder est accessible et constitue un lieu idéal pour l'observation des oiseaux et pour la chasse à la sauvagine en pourvoirie.

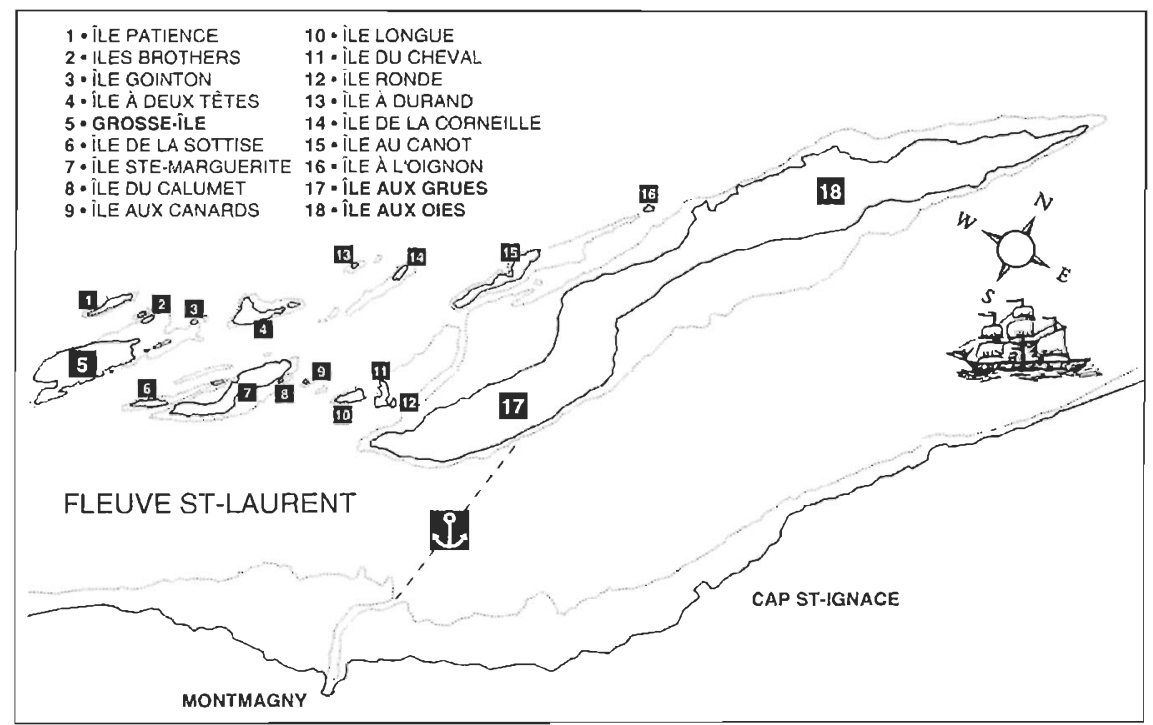

Figure 1. L'archipel de l'Île aux Grues (Vézina 1994 : 4).

7. Les chiffres pour la population varient de 110 (Excursion à Isle-aux-Grues. Entre temps et marées, 2006), 125 (Ruel 2006), 154 (Tourisme ChaudièreAppalaches et Tourisme Québec 2006: 83), à 159 (Bouchard 2004 : 156). 


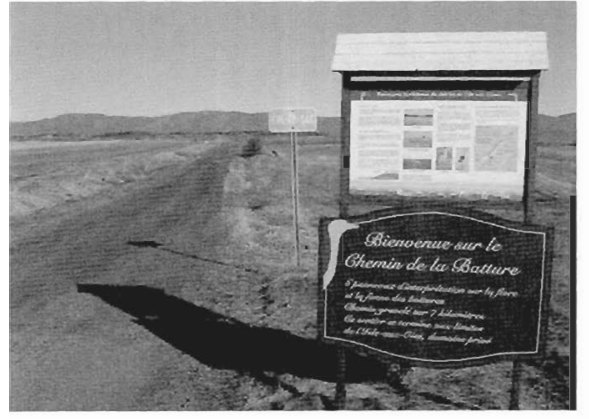

Figure 2. Début du chemin de la Batture (Huot 2006).

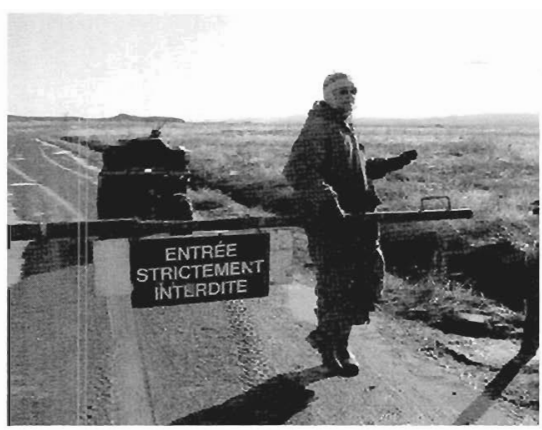

Figure 3. Barrières interdisant l'accès à l'Île aux Oies (Huot 2006).

Le contexte insulaire est très marqué à l'île aux Grues, principalement par son accès restreint. D'abord, la société des traversiers du Québec offre ses services entre l'île aux Grues et Montmagny seulement des mois d'avril à décembre, après et avant la formation des glaces sur le fleuve Saint-Laurent. Par ailleurs, la traversée n'est possible qu'en tenant compte des marées. Ainsi, certaines journées ne permettent qu'un voyage et le nombre de places sur le traversier est limité. Il faut aussi savoir que le traversier "couche " toujours à l'île; une fois amarré, il ne revient à Montmagny que si les conditions météorologiques et la marée lui permettent de revenir à son port d'attache. La population de l'île doit donc quotidiennement s'adapter à son contexte naturel, à moins d'emprunter une liaison aérienne, plus régulière, qui existe depuis 1954. Aujourd'hui, un fait demeure manifeste : la relation avec le continent s'effectue plus aisément par avion, avec un certain confort et un effort moindre, comparé au temps de la traverse en canots.

L'autarcie est une notion de plus en plus utopique dans la société contemporaine, mais il n'en demeure pas moins que l'existence d'une population sur une île dépend principalement d'une autonomie, d'une indépendance économique. Elle doit pouvoir produire et exploiter ses ressources à même son territoire et, si besoin est, offrir des services à l'extérieur. Pour sa part, l'Île aux Grues vit principalement de trois secteurs économiques, soit la production laitière et fromagère, le tourisme et la chasse. 
L'économie principale de l'île, c'est certain que ça tourne beaucoup autour de la fromagerie, parce que les cinq producteurs laitiers de l'île qui restent maintenant produisent en coopération pour la fromagerie. Donc, tout le lait qui est produit là s'en va directement à la fromagerie.... Après ça, oui, le tourisme l'été apporte une économie assez importante pour une l'île qui n'a que cent dix habitants seulement maintenant (Amélie Ringuet).

À la suite du tourisme s'ajoute la chasse. Si la belle saison, qui débute officiellement le 20 mai et va jusqu'au 30 septembre ${ }^{8}$, comme bien des endroits au Québec, est le moment fort pour le tourisme de plaisance, l'automne apporte un tourisme différent, qui vient presque uniquement pour la chasse. "En octobre, on commence la période de la chasse, alors c'est une autre sorte de touristes, c'est des chasseurs. Ils vont sur les berges toute la journée.... Alors, ce n'est pas rentable de garder le musée ouvert "(Gina Vézina). Pour les chasseurs qui vont sur l'île, l'intérêt ne se situe pas au niveau touristique, puisqu'ils ne se définissent pas sous ce vocable, mais bien sous celui de chasseur : "Non, on n'est pas allés en tant que touriste, on est allés en tant que chasseur » (Clément Belley). Le magnétisme qu'exerce l'archipel sur la chasse et l'abondance du gibier sont en lien aussi avec la privatisation accrue de ces îles, notamment par des amateurs américains, et la valeur de cellesci est fixée en fonction de l'abondance du gibier. "Aucune industrie, aucun projet domiciliaire, aucun centre de villégiature; que des cultivateurs et des chasseurs, les premiers hébergeant les seconds " (Croteau 1995: 95). Enfin, la combinaison de ces trois secteurs économiques "permet aux insulaires restants de pouvoir vivre et demeurer dans l'archipel. La perte d'une seule de ces trois activités aurait des conséquences sur les autres. La plupart des agriculteurs et des hôteliers ont besoin de la chasse pour survivre et le tourisme est un outil indispensable à la bonne marche de l'économie locale " (Vézina 1994: 132-133). En ce sens, le tourisme peut assurer un nouveau développement et amener un bassin potentiel de consommateurs.

\section{La construction d'un tourisme culturel : les attraits touristiques}

Parmi les attractions à étudier, la première est sans doute la traversée

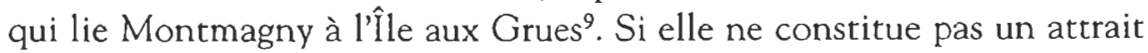
au sens propre du terme, elle n'en demeure pas moins une étape quasi

8. Période où il est possible de visiter les attraits touristiques de l'île.

9. Traversée d'une durée de vingt-cinq minutes. 
incontournable pour le visiteur qui désire atteindre l'île. En fait, à elle seule, elle marque la coupure avec le continent. En effet, les insulaires, tout comme les touristes, doivent utiliser principalement le traversier de la société des traversiers du Québec, un service gratuit, mais qui n'est pas offert à heure fixe. Le premier défi est d'informer le touriste que ce sont les éléments de la nature et principalement la marée, qui fixeront l'horaire de sa journée. "Il faut qu'il soit conscient aussi que ce n'est pas la traverse Québec-Lévis, où il y a des départs l'été presque toutes les trente minutes. Ici, on a la problématique des marées» (Amélie Ringuet). De même, nul n'a établi de pont entre l'île et $M$ on tm agny, conservant ainsi in tact le caractère un ique de lîlle ${ }^{10}$. En lien avec la problématique liée à la traversée, l'île acquiert une plus value au plan de l'imaginaire. Ajoutons l'arrêt du service pendant l'hiver (figure 4) et le dépaysement est maximisé, "vu qu'il n'y a pas de traversée en bateau l'hiver, ben ça donne un cachet, ça donne une certaine attraction à l'île " (Jean Gosselin).

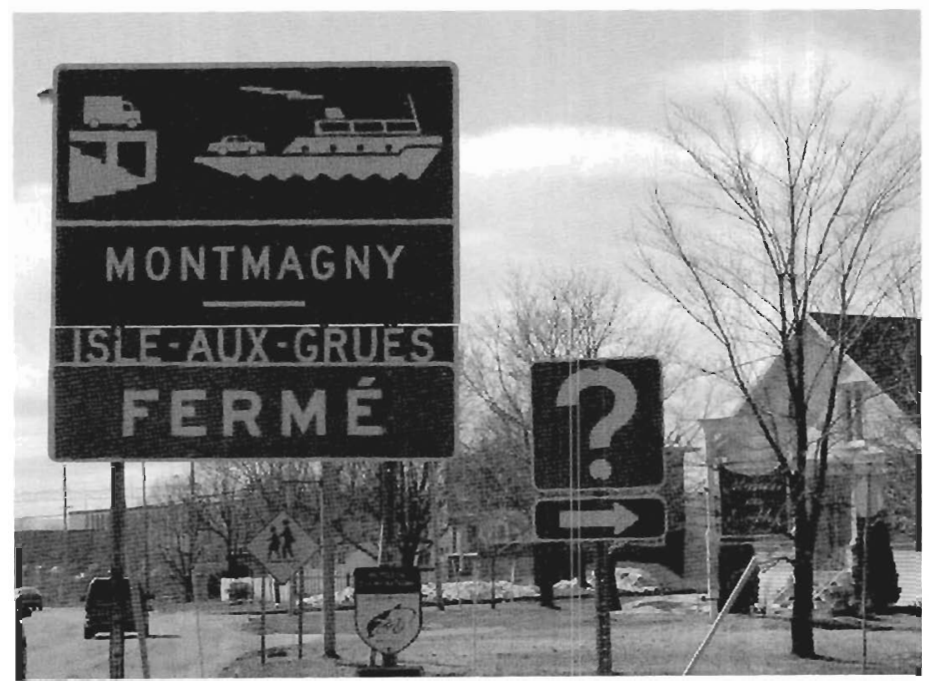

Figure 4. Indication de la fermeture de la traverse pour l'hiver (Huot 2006).

10. Pensons à l'île d'Orléans qui est reliée par un pont à la côte de Beaupré depuis 1935 : le caractère insulaire est préservé, mais l'expérience de traverser n'est plus sujet à un dépaysement et le touriste n'est plus enfermé dans un horaire précis imposé par un traversier, il peut choisir l'heure de son arrivée et de son retour. 
Quand les gens arrivent sur l'île, après leur traversée, ils ont la possibilité de monter à bord d'un train-balade géré par la corporation touristique de l'Île-aux-Grues. Celui-ci offre quatre-vingt places réparties dans quatre wagons et amène les visiteurs au centre du village. Cet attrait est particulièrement apprécié par les personnes qui ont de la difficulté à se déplacer : "C'est fin ça pour les personnes âgées" (Bernadette Leblond). Le circuit de la visite guidée comprend l'interprétation par un guide engagé par la corporation touristique des principaux attraits de l'île. Le train les amène au Musée Le Grenier de l'Isle (figure 5), qui comprend une salle d'exposition où l'on retrouve différents éléments, témoins de l'art et de l'histoire de l'île, ainsi qu'une salle de documentation portant entre autres sur le peintre Jean-Paul Riopelle. Une halte est également effectuée au Centre de la Volière (figure 6) qui présente une exposition sur la tradition de " $\mathrm{La} \mathrm{Mi-}$ Carême ", où sont exposés des costumes de célébration dont le costume traditionnel des galonnés (figure 7), ainsi que des expositions sur «Les artistes gruois " qui regroupent des œuvres des artistes locaux. Il existe un passeport qui permet aux gens de visiter les deux musées, ainsi que l'église, datant de 1888 (figure 8). Il y a aussi un arrêt à la Fromagerie de l'Isle-aux-Grues (figure 9), coopérative agricole, où sont mis en évidence des traits importants de l'île par le nom de certains fromages artisanaux, comme la Mi-Carême et le Riopelle. "Le célèbre artiste a donc accepté de céder son nom, de même que l'œuvre Mi-Carême pour l'emballage du produit, peint à l'île aux Grues en 1990 " (Fernandez 2002c : 2). Or, cet attrait mis de l'avant par les promoteurs touristiques fait d'une certaine manière chou blanc. En effet, les visiteurs ont des attentes et ils aimeraient observer de plus près la fabrication de ces fromages. "Ça, c'est quelque chose que l'on se fait demander : "Est-ce qu'on peut visiter la fromagerie?" (Amélie Ringuet). Seul un comptoir des ventes est accessible aux visiteurs.

Au niveau de la restauration, les touristes ont aussi la possibilité de se régaler au Bateau Ivre" (figure 10), ancien remorqueur, "amarré pour ses derniers jours" (Bouchard 2004: 158), qui a été converti en salle à manger. Il offre aux touristes plaisir gustatif et visuel avec vue sur l'archipel, en plus d'accueillir ses invités avec les vers du poème d'Arthur Rimbaud, "Ô que ma quille éclate! Ô que j'aille à la mer! »

11. L'origine de cetre appellation serait attribuable à Marthe Belleau, mère de Jean O'Neil. L'auteur relate l'anecdote dans son livre consacré à l'île aux Grues (1991: 177-180). 


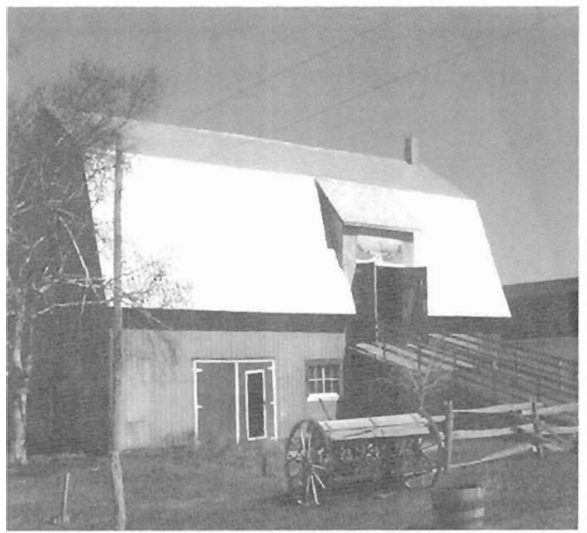

Figure 5. Le Musée Le Grenier de L'Isle (Huot 2006).

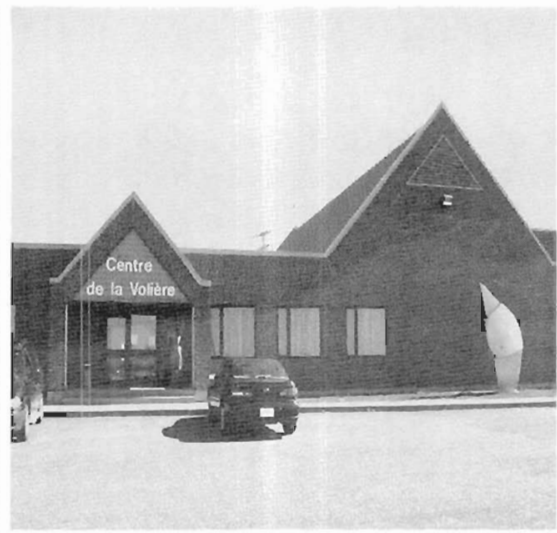

Figure 6. Le Centre de la Volière (Huot 2006).

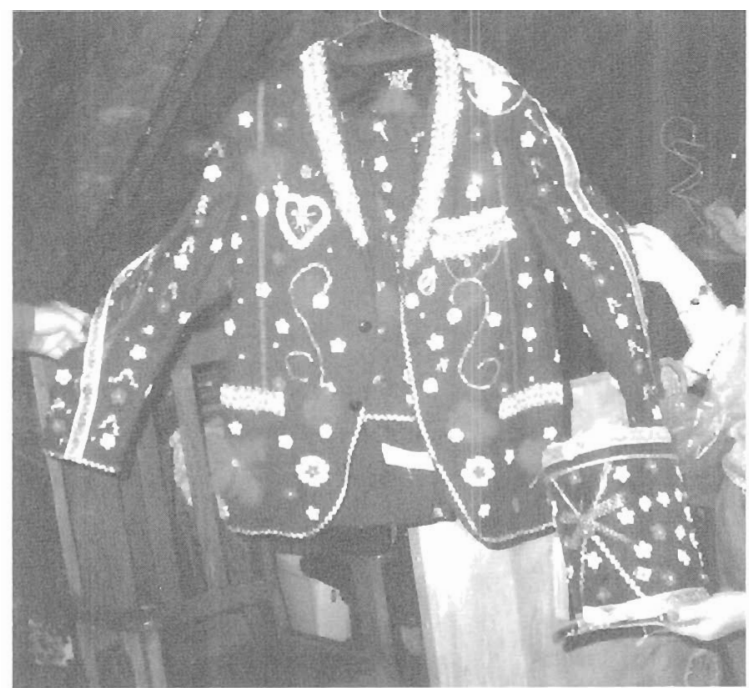

Figure 7. Le costume du Galonné est un complet noir ou marine décoré de rubans multicolores, de paillettes, de fleurs de papier, de plumes et assorti d'un masque et d'un chapeau en forme de mitre d'évêque (Roberge 1993). 


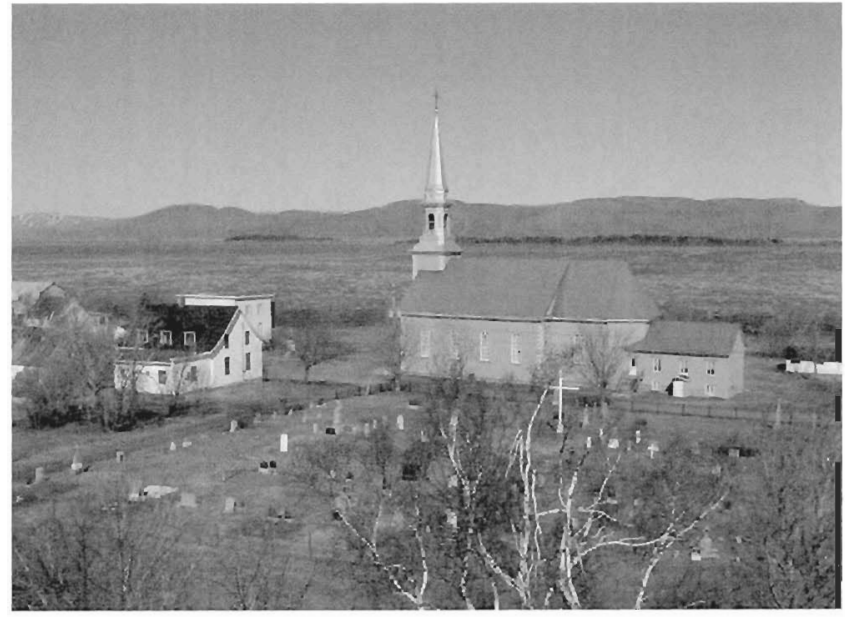

Figure 8. Vue du cimetière et de l'église (Huot 2006).

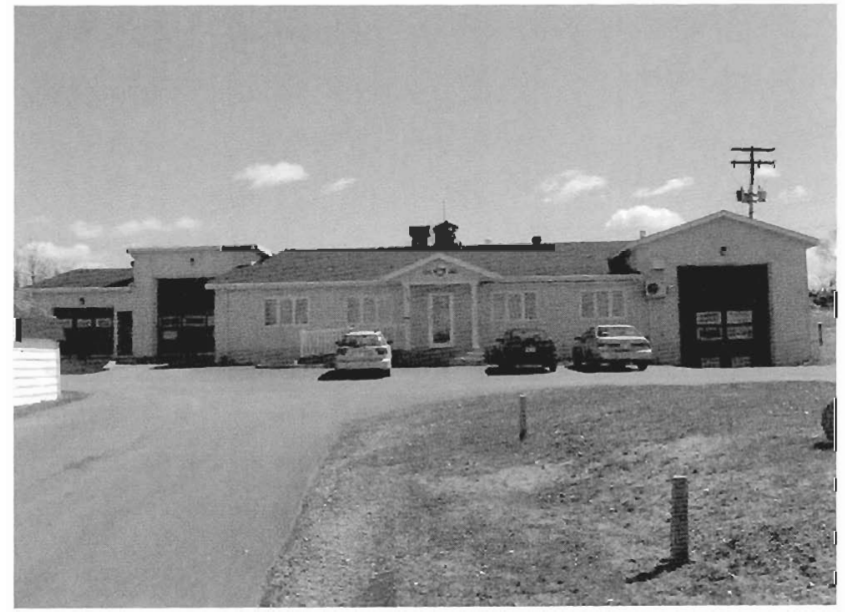

Figure 9. Façade de la fromagerie (Huot 2006). 
(1992 : 71). Cette salle à manger, par son allure unique, figure dans les attraits suggérés sur l'île (Ruel 1997; Stanton 1999). À la liste des points à mettre dans la mire des touristes, les promoteurs mentionnent aussi le Manoir seigneurial McPherson-Lemoyne (figure 11). Ce manoir historique a vu ses fonctions changer au cours des années, de manoir seigneurial à bed and breakfast, il est maintenant une résidence privée depuis 1995. L'association touristique de l'île suggère tout de même de lancer un coup d'œil sur son architecture à travers les barreaux de sa clôture. Bien que le nom du nouveau propriétaire ne soit pas mentionné dans son dépliant touristique, certains guides touristiques présentent tout de même le manoir comme étant la résidence du peintre JeanPaul Riopelle (Rémillard 2003 : 507), conférant ainsi au lieu une aura internationale par la renommée de l'artiste.

Enfin, l'île est également un site connu et reconnu pour les ornithologues, car on y retrouve plus de deux cents types d'oiseaux. Ornitour offre d'ailleurs des visites ornithologiques guidées sur l'île. Fin mai, il y a une fin de semaine consacrée à l'ornithologie. L'̂̂le complète ses activités en proposant trois sentiers pédestres: Sentier de la Grève et de la pointe-aux-Pins $(4.5 \mathrm{~km})$, Sentier de l'Érablière $(1.6 \mathrm{~km})$ ainsi que Chemin de la batture $(6 \mathrm{~km})$. Il y a aussi les croisières privées, dont les Lachance de Berthier-sur-mer, qui offrent un circuit nature/culture, avec un guide sur l'île.

En somme, ces attractions touristiques ne misent pas uniquement sur la promotion d'une identité insulaire, bien que celles-ci soient liées à leur milieu. Ainsi, la traverse suit le cours des marées. Et si l'on trouve des fromageries à travers tout le Québec, le fromage de l'île tire sa spécificité de son milieu, avec des noms évocateurs tels que le $\mathrm{Mi}$ Carême, le Riopelle et le Tomme de Grosse-île. Les deux musées exposent l'art et l'histoire de l'île. Quant au restaurant situé dans une épave échouée, il met en évidence les vestiges maritimes de l'île. Mais pour déterminer si la mise en valeur du tourisme est intimement liée à l'identité et au mode de vie des insulaires, il faut tenter de dégager les manifestations qui lui sont propres. 


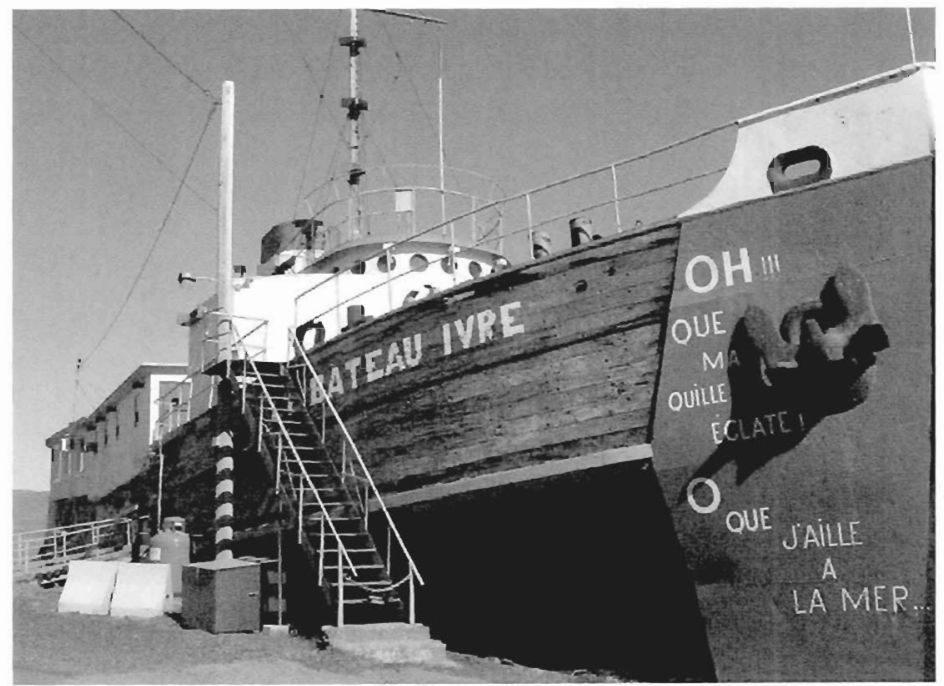

Figure 10. Le Bateau Ivre, entrée pour monter à bord (Huot 2006).

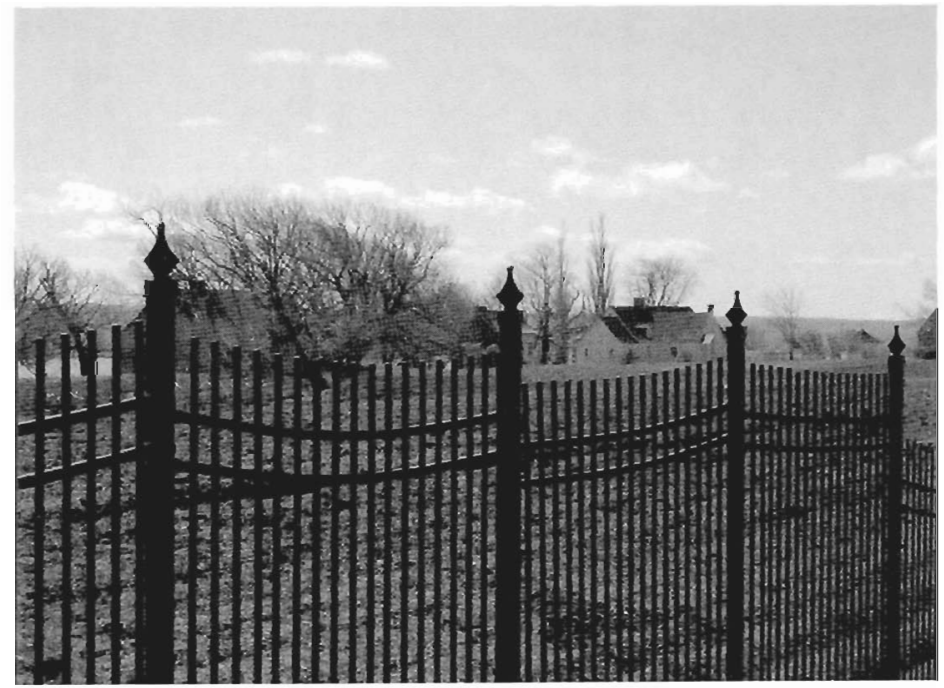

Figure 11. Vue du Manoir McPherson-Lemoyne, résidence de Jean-Paul Riopelle (Huot 2006). 


\section{Les manlfestations propres à l'île aux Grues}

\section{Lami-carême}

Au cours de l'histoire de leur implantation à l'île aux Grues, les insulaires ont dû développer des activités pour rendre leur solitude moins lourde et moins contraignante. Ainsi, quand le traversier cesse, que les glaces isolent les îles, "[les insulaires] répondent au froid, à l'hiver et à leur isolement avec les moyens de fortune ... les canots d'hiver, le corne-en-cul, l'encan des âmes, les veillées, le whisky de contrebande ... et la mi-carême "(Perrault 1964: 26). La tradition de la mi-carême est célébrée ${ }^{12}$ vers la deuxième semaine de mars ${ }^{13}$, bien que dans une forme actualisée ${ }^{14}$, où " les comportements contemporains se greffent sur les rituels anciens"(Du Berger 2001:9). Cette fête consiste à passer de maison en maison en petits groupes et déguisés. Les mi-carêmes changent leurs voix, leurs démarches et leurs mimiques afin de ne pas se faire reconnaître et mystifier les hôtes qui essaient alors de découvrir qui se cache derrière le masque. La veillée se poursuit ainsi, accompagnée d'airs joués au violon et à l'accordéon. Au fil des ans, les costumes sont devenus de plus en plus sophistiqués et si, dans un premier temps, chaque membre de la fête fabriquait son déguisement, les couturières de l'île confectionnent aujourd'hui un costume nouveau chaque année autour d'un thème ${ }^{15}$. Ce "véritable spectacle populaire" (Landry 2001: 20) permet de rompre avec l'hiver ${ }^{16}$, le quotidien, l'isolement. "C'est sûr que, pour les gens de l'île, c'est un événement important pendant l'hiver parce que justement, ça brise peut-être un peu l'isolement »(Amélie Ringuet).

La tradition de la mi-carême, qui a aidé à populariser l'île, fait de celle-ci un endroit sollicité par le touriste ${ }^{17}$. L'île est alors perçue en

12. De nos jours, il ne reste que trois villages québécois qui célèbrent encore cette fête : Natashquan, sur la Côte-Nord, Fatima, aux Îles-de-la-Madeleine et l'Île aux Grues. La fête est célébrée également à Chéticamp, en Acadie.

13. La célébration de la mi-carême est tributaire de la fête de Pâques.

14. La coutume est interrompue dans les années 1950, pour être reprise en 1975 (Landry 2001: 22).

15. Auparavant seulement les hommes couraient la mi-carême; aujourd'hui, femmes et enfants se costument.

16. Bien que la confection des costumes rompe avec l'hiver, les couturières s'y affairent dès l'automne.

17. La présence des touristes est toutefois un aspect très récent de la fête. 
tant que lieu-réceptacle ${ }^{18}$ d'une identité à conserver. La publicité exercée par les reportages journalistiques et télévisuels a contribué à la séduction d'un bon nombre de touristes qui veulent prendre part à la fête. De même, les habitants de l'île communiquent aux touristes les particularités de cette célébration qui survit au passage du temps:

Les gens qui sont venus l'été, qui ont passé plus qu'une journée, ils ont le temps de se faire raconter ça. Exemple, des gens qui vont ĉtre dans un gîte ou dans une auberge, ils ont le temps d'avoir des dialogues avec les gens, les insulaires, puis de se faire raconter comment ça se passe, de voir des vidéos, plus qu'une fois parce que c'est exposé làbas les costumes de la mi-carême. En saison, ils ont le temps d'y aller une fois ou deux, ils ont le temps de voir ça, puis là, ça a le temps de leur donner la piqûre de revenir au mois de mars (Gina Vézina).

Par contre, cette affluence touristique ne va pas sans heurt, "pour ce qui est du niveau touristique, ils ont une capacité d'accueil quand même. Parce que cette année, il y a eu deux cent cinquante personnes pendant la fin de semaine de la mi-carême. Mais c'est certain, il ne pourrait pas y avoir six cent personnes parce que les infrastructures sur l'île ne sont pas suffisantes pour accueillir tous ces gens-là " (Amélie Ringuet). Cette capacité d'accueil est un frein au développement de l'activité touristique : les gîtes et auberges sont remplis à pleine capacité et seul le service aérien assure la traversée à l'île. En somme, assister à la mi-carême est un privilège ${ }^{19}$. "Durant cette période-là, il y a beaucoup de touristes. Durant une fin de semaine, c'est une grosse fin de semaine à cause on peut voyager dans une journée tout près de deux cents personnes " (Jean Gosselin). Toutefois, le touriste qui assiste doit être conscient de son rôle restreint, il est confiné à celui de spectateur. Le touriste ne participe pas à la fête, il ne peut que l'observer. En effet, lors de la mi-carême, un touriste ne peut se déguiser et s'attendre à être reconnu. De même, il ne peut reconnaître les insulaires qui lui sont étrangers. Sa participation réduirait à néant les tenants de la célébration. Or, cela n'est pas nécessairement pour déplaire. En fait, le touriste se définit lui-même bien plus souvent comme un voyeur puisque "le touriste d'aujourd'hui serait séduit par le caractère factice de son expérience, plus en quête de divertissements que de traditions

18. Ce terme de lieu-réceptacle est emprunté à Picard 1992.

19. Les touristes ne peuvent participer qu'à la soirée à la salle communautaire; ils ne vont pas dans les maisons. 
authentiques» (Le Menestrel 2002: 469). Et de quelque côté que l'on soit, ce rôle semble celui qui convient le mieux. L'expérience touristique de la mi-carême dure une fin de semaine au bénéfice des touristes qui viennent s'y divertir alors que les festivités retrouvent leur authenticité pour la population locale qui poursuit la fête une semaine durant.

\section{La troversée en canot à glace}

Afin de mettre à mal l'éloignement et l'isolation de l'île, une autre innovation a été mise de l'avant par des insulaires. "Le canot à glace est le fétiche numéro deux de l'île, après les oies, bien sûr " (O'Neil 1991 : 44). Ce moyen de transport hivernal a traversé une période de l'histoire des insulaires en marquant fortement leur identité insulaire. Autrefois, un équipage de canotiers pouvait se lancer sur un fleuve gelé pour aller chercher le médecin, comme en a témoigné le Docteur Joseph Cloutier dans le récit d'une traversée qui eut lieu le 26 janvier vers 1925 entre Cap-St-Ignace et l'Île aux Grues (cité dans Richard $1971: 315-3222^{20}$. C'était un moyen de transport absolument nécessaire comme le souligne Joseph Lachance fils, originaire de l'Île au Canot, " les canots ... c'était ni plus ni moins que nos taxis " (cité par Lavoie 2001 : 32). Le site Internet de la Corporation de développement touristique de L'Isle-aux-Grues met l'accent notamment sur cette pratique comme faisant partie de la vie insulaire d'antan.

Mais en 1954, le premier service aérien régulier débute et remplace progressivement le canot à glace. "On n'a plus besoin de ça. Avec l'avion, avec une infirmière sur l'île, on peut toujours parer aux urgences" (O'Neil 1991: 45). L'apparition du service aérien va changer la vie des insulaires. L'île entre dans une ère de modernité qui affecte le mode de vie des habitants : "La tradition s'est arrêtée à cause de la modernisation "(Amélie Ringuet). Cependant, l'utilisation du service aérien chez les insulaires a été freinée par son coût avant d'être le moyen de transport généralisé pour toute la population, comme le nuance Gina Vézina, car pour les gens, "c'était un luxe de prendre l'avion. Les gens ne le prenaient pas, ils prenaient le canot. Tu sais, maintenant, on prend l'avion aussi souvent que l'on veut, ça coûte vingt dollars allerretour pour les insulaires". Elle précise également que "maintenant l'avion vient deux, trois, quatre, cinq fois par jour. Et puis, ça fait partie

20. Ce texte est également repris dans Lemieux (1978: 136-144), Deschênes (2001 : 312-318), ainsi que dans Croteau, dans une version non intégrale (1995: 8489). 
de la vie des insulaires. Mais si tu remontes à trente ou quarante ans, bien non, le canot à glace il allait trois fois par semaine à Montmagny pour rapporter les marchandises, l'épicerie, le courrier et tout ». Autre facteur non négligeable dans la disparition de la tradition : la désertion des îles voisines et leur privatisation entraînent ainsi la fin du cabotage d'hiver. Autrefois, les gens voyageaient dans les îles avec les canots, car tout ce territoire était le leur et ils se l'appropriaient. Aujourd'hui, ils sont de plus en plus confinés sur leur île au milieu de l'archipel et l'unique relève pour cette activité, ce sont les gens qui y trouvent un intérêt sportif.

Cette pratique culturelle n'est pas reprise à des fins touristiques par les gens de l'île. Or, l'activité sportive persiste en dehors de l'île, car elle a été incluse dans les activités du premier carnaval d'hiver de Québec en 1894, par une course entre Lévis et le bassin Louise. En 1955, après un interlude de quelques années, "le carnaval de Québec, véhicule privilégié de la rusticité et du pittoresque, intègre à sa programmation cette activité navale typique du Québec qui deviendra une classique " (Lavoie 2001: 33). Si l'île aux Grues a effectué la transition vers un autre mode de transport, la traversée en canot d'hiver demeure toujours vivante par la course sportive.

Symbole d'insularité par excellence, la traversée en canot à glace demeure une pratique qui n'est pas totalement abandonnée par les insulaires, qui font des équipes pour courser au carnaval d'hiver de Québec. Ils conservent tout de même en mémoire l'inventivité et la force des anciens: Le Grenier de l'Isle est un musée qui expose les canots à glace. En ce sens, cet attrait ne prétend pas figer les insulaires dans une façon de vivre révolue, mais rend hommage à une pratique liée à l'insularité.

La seconde [approche] s'attache aux dimensions culturelles du tourisme et n'envisage pas celui-ci seulement comme un instrument de destruction, mais comme un facteur potentiel de création : la réification de la culture locale via le tourisme ne détruit pas toujours celui-ci ... (Ramos 1999: 289).

La mise en place d'une structure vouée au tourisme, une institution muséale, permet plutôt de conserver la trace de la culture insulaire. L'objet muséologique acquiert ainsi le statut recherché par les touristes, l'objet devient le témoin, l'aura d'une pratique conservée, la trace d'un passage. 
Jean-Paul Riopelle

Certes, le peintre Jean-Paul Riopelle n'est pas une manifestation propre et issue de l'insularité. Pourtant, en adoptant comme lieu de résidence l'Île aux Grues pour son isolement et ses oies, il prenait part à cette insularité et devenait objet de convoitise des touristes.

Né à Montréal en octobre 1923, Jean-Paul Riopelle décède à l'Île aux Grues en mars 2002, à l'âge de 78 ans. Élève de Paul-Émile Borduas à l'École du meuble, signataire en 1948 du Refus global, il s'installe à la fin de 1948 à Paris ${ }^{21}$. Passant de l'aventure automatiste à Montréal à un paysagisme abstrait à Paris, il fréquente André Breton et le mouvement surréaliste, qu'il abandonne rapidement. Ami de Samuel Becket, Antonin Artaud, Alberto Giacometti, Juan Mirò et amant de Joan Mitchell pendant près de vingt-cinq ans, son rayonnement international lui permet de toucher divers médiums : peinture, sculpture, installations, estampes, dessins. Il demeure pendant quarante ans en France. En 1989, il revient au Québec et dès 1991, il se procure une maison sur l'île aux Oies (figure 12), où il y aménage un atelier. Le peintre continue à approfondir son art, allant même jusqu'à surprendre la critique en abandonnant l'abstraction pour retourner à la figuration des années 1970 , donnant à sa peinture au fil du temps, un nouveau vocabulaire avec un bestiaire aux teintes de l'île où il demeure (des oies, des grues ... $)^{22}$. Vers 1993, il interrompt son travail, miné par l'ostéoporose.

En 1995, il acquiert le Manoir ancestral McPherson et devient finalement un insulaire d'adoption, comme en témoigne Riopelle luimême : "Il y a plus de 30 ans que je viens ici à la chasse. Sur les îles de l'archipel de Montmagny, je connais tout le monde et tout le monde me connaît. Les habitants ne me considèrent pas comme quelqu'un qui vient du "continent " mais bien comme un insulaire" (Viau 2002: Z1). Les propos de l'artiste sont corroborés par les insulaires, "il faisait partie de la vie des insulaires"(Gina Vézina). Ceux-ci lui accordent une importance significative pour l'île, "On a été chanceux de l'avoir avec nous. Cela a mis l'île sur la carte", a conclu Louise Dion-Roy ${ }^{23}$, qui ne sait pas encore de quelle façon les insulaires honoreront leur célèbre concitoyen d'adoption" (Fernandez 2002a : 3). Le peintre s'est

21. Il avait déjà été à Paris en 1946.

22. Dans le présent article, je n'analyse pas l'œuvre picturale du peintre, celle-ci n'étant pas mise de l'avant dans la promotion touristique de l'île aux Grues.

23. Louise Dion-Roy était mairesse de l'île aux Grues à l'époque du décès du peintre. 


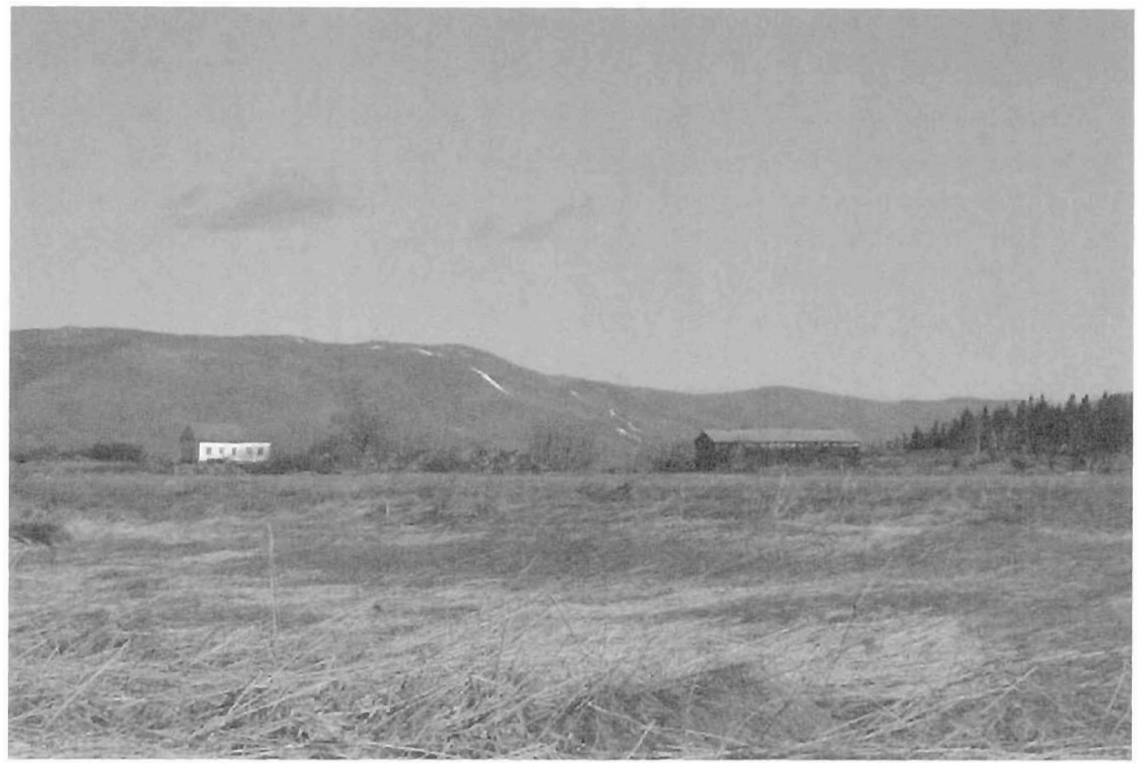

Figure 12. Maison-atelier de Jean-Paul Riopelle à l'Île aux Oies (Huot 2006).

même vu personnifier par des mi-carêmes, "un farceur qui aura pris les traits de Riopelle " (Ruel 1996: 44). La présence de Jean-Paul Riopelle sur l'île aura fait couler beaucoup d'encre. À savoir s'il a fait l'unanimité chez les insulaires, certaines personnes soutiennent que non. MarieHélène Comeau évoque une controverse concernant l'acquisition du manoir par le peintre.

La vie s'écoulait paisiblement, lorsqu'un peintre de grande renommée décida d'y poser ses pinceaux à l'aube ${ }^{24}$ de sa vie. Il s'agissait là d'un grand bouleversement, car, une fois prise, cette décision entraîna l'acquisition de la plus belle, de la plus grande demeure des lieux : le manoir de l'île. Lieu prestigieux que les habitants de l'Ile-Aux-Grues s'étaient approprié dans leurs habitudes quotidiennes. Ainsi, lors des belles journées d'été par exemple, les gens prenaient plaisir à faire visiter cet endroit à leur parenté venue de loin. Quel bonheur également de se prélasser dans la grande balançoire du manoir en écoutant le chant du fleuve. Mais, une fois achetés, les lieux devinrent la chasse gardée de l'artiste peintre.

24. La figure de style ne semble pas adéquate. Il aurait été plus juste d'utiliser "crépuscule». 
Finie, donc, la belle époque des promenades au manoir. Cette décision fit l'effet d'une bombe sur l'île. Les estuaires [sic] se voyaient dépossédés de leur manoir et, par le fait même, de leur balançoire. La paix sociale des jours heureux était brisée. L'artiste flamboyant se nommait JeanPaul Riopelle. Cette histoire a été recueillie lors d'un séjour dans la ville de Montmagny, située sur les côtes, juste en face de l'Ile-AuxGrues. Ainsi, pour certains, le nom de Riopelle restera dans les mémoires comme celui du briseur de l'harmonie qui régnait sur l'île (Comeau).

Cependant, sa présence a aussi contribué à une certaine affluence touristique. Il y a une clientèle qui désire observer ces lieux où a vécu le peintre, où il trouva l'inspiration pour ses célèbres oies. Mais en définitive, pour le touriste, l'homme est plus significatif que son œuvre. "Pourtant, ce n'est pas tant l'œuvre qui fascine le public, mais bien le personnage" (Bernier 2002: 12). Bien que le peintre ne soit pas mis de l'avant dans la promotion touristique (dépliants et brochures), il demeure un attrait touristique important pour la clientèle qui visite l'île, "aussitôt que c'est médiatisé, regarde, il y a des gens qui veulent savoir " (Johanne Ouellet). L'attraction du peintre est réelle et ce, malgré son décès et l'impossibilité de visiter sa demeure. Les gens, encore une fois, demeurent spectateurs. Ils laissent plutôt vibrer dans leur imagination le spectacle du peintre qui a investi l'imaginaire collectif. "Personnage de légende " (Bernier 2002 : 12) qui " a contribué à rendre célèbre son île» (Carignan 2002: Z3), sa demeure devient pour le touriste un lieu important : "Là, je sais où qu'il est, moi. C'est ça, c'est de savoir quand j'ai quelqu'un que je connais, quelqu'un d'important, c'est d'aller voir sur les lieux où il a vécu " (Bernadette Leblond). Une telle manifestation n'est pas unique; plusieurs autres sites ont bénéficié de telle retombée:

Comme Félix, Félix Leclerc à l'île d'Orléans, c'est comme SaintePétronille pour les petits Simard à l'Île d'Orléans aussi, l'Île Verte, Gilles Carle, malgré que c'est tellement ... J'pense que c'est tellement tellement tranquille. Puis j'pense que les gens doivent être hyper respectueux. Tu sais, ce n'est pas la place où tu te garroches à plat ventre. Moi, j'ai déjà vécu ça parce que je connais très bien Rock Voisine. Il restait dans mon village et dans la rue où je restais. Et j'ai été élevée avec lui. Puis je travaillais dans un commerce, une auberge, moi, une auberge réputée, puis écoute, quand Rock est devenu très populaire, je te le dis, les gens qui venaient, les Français, venaient passer des étés chez nous. Puis des semaines juste pour le voir, tu sais. Tu te dis ça doit coûter un bras venir icitte (Johanne Ouellet). 
"Quand [Riopelle] était en vie, oui, ça a augmenté l'achalandage " (Jean Gosselin). Mais l'essor touristique destiné au peintre s'est fait réellement sentir à son décès, comme l'explique Gina Vézina: «L'impact, ça a été le décès de monsieur Riopelle, l'été qui a suivi, les gens ont débarqué sans bon sens, en pensant qu'ils pourraient aller au Manoir McPherson ou en pensant qu'ils pourraient visiter ses affaires ou quoi que ce soit. Mais non, c'est privé ". L'été du décès du peintre, l'île a vu sa terre investie d'une forte demande: "Quand il est décédé, ça a amené une affluence touristique plus élevée, parce que les gens en ont tellement entendu parler de Riopelle à la télé pendant cette période. là que là, les gens voulaient aller voir où il habitait. Ça fait qu'il y a eu une grosse affluence" (Amélie Ringuet). Elle précise même que, pour certains touristes, c'était le but de leur présence, car les visiteurs venaient exactement pour cette raison : rendre hommage à l'artiste. Les touristes trouvent parfois eux-mêmes les chemins de leurs désirs. Cependant, dans le cas présent, ils sont plus souvent déçus: l'artiste et sa demeure ne sont pas des «objets" touristiques. La frontière est décelée, celle où l'individualité, le privé, entre en confrontation avec le touriste, spectateur impénitent. À l'opposé, ce type de tourisme peut-il avoir un impact néfaste sur la culture locale?

La promotion touristique et ses impacts : le tourisme culturel et les attentes

"Pendant la saison estivale, de plus en plus de touristes défilent sur les petites routes tranquilles, visitent et puis s'en vont " (Bornais 2003b: 42). De cette manne de voyageurs, l'on peut dire que l'île sait charmer différents types de touristes: "En juillet, c'est des petites familles; puis en août, c'est une autre sorte de touristes aussi; puis en septembre, c'est des personnes un peu plus retraitées"(Gina Vézina). Le tourisme représente donc un facteur de développement économique important, non seulement sur l'île aux Grues, mais cet impact rejoint de près ou de loin toute la région de Montmagny. L'intérêt est majeur :

Les gens, quand y viennent, ça peut être leur destination l'île aux Grues parce que ce qui arrive, c'est que la région ici, en étant à proximité de Québec, on est une région où l'on a beaucoup d'excursionnistes ${ }^{25}$, donc souvent les gens vont pas passer trois, quatre, cinq, six nuitées ici, comme on fait si on allait à Montréal, comme on

25. Par excursionniste, l'on entend ici une personne qui vient passer une journée sur l'île, mais qui n'y séjournera pas pour une nuit ou plus. 
fait si on allait à Québec puis que l'on venait de la Gaspésie puis on allait à Québec. Les gens vont passer plusieurs jours souvent. Tandis que là, en étant à proximité des grands centres, les gens vont plus faire des excursions. Ça fait que pour les gens de Québec, c'est facile quand tu as l'horaire du traversier puis ça adonne. Les gens viennent, laissent leur voiture ici, partent en vélo, vont faire le tour en vélo à l'île (Amélie Ringuet).

Mais si tous ces touristes choisissent l'île, l'on peut se demander en quoi elle répond à leurs attentes, ce qu'elle éveille comme attraction.

Dans le tourisme culturel mis en œuvre en milieu insulaire, le visiteur serait-il à la recherche d'une intimité, d'un mode de vie qui témoigne d'un temps révolu, d'un autre sens à la vie contemporaine? "Les insulaires ont une mentalité particulière, puis on aime ça. On aime ça tu sais, la pensée de ces gens là" (Johanne Ouellet). Parmi les gens interrogés, les caractéristiques les plus citées qui stimulent le désir de découvrir l'île, c'est d'abord un environnement autre, "la senteur de l'environnement, parce que ça ne sent pas comme chez nous. C'est l'air, ça a une odeur, la senteur, j'aime ça " (Bernadette Leblond); puis la possibilité de se reposer, "tout est ralenti là-bas, la vie c'est plaisant » (Johanne Ouellet) ; et surtout, l'attrait de la différence, "la curiosité, ... j'aime ça voyager, aller voir autre chose que ce qu'il y a par chez nous " (Bernadette Leblond), " une curiosité pour les gens de voir comment les insulaires vivent "(Amélie Ringuet), "la vie des gens de l'île, les insulaires, c'est quelque chose que tu revois pas ailleurs. Tu vas aller sur l'île, les gens, les insulaires, c'est des gens qui ne sont pas à la course comme nous autres. Tu sais, nous autres, bing bang arrive quelque chose puis on repart, tandis que les insulaires prennent le temps, plus le temps de vivre un peu. C'est l'espèce d'isolement qu'il y a sur l'île, la quiétude, la beauté de la nature, c'est un peu ça qu'ils recherchent "(Jean Gosselin).

Cependant, ces attentes ne peuvent toujours se réaliser. Et la confrontation avec l'Île aux Grues d'aujourd'hui amène les gens à faire face à la réalité. "Il y a eu un certain engouement pour l'île ces dernières années, puis peut-être que les gens se sont fait un peu trop d'attentes » (Jean Gosselin). Aussi, il est primordial pour éviter les insatisfactions que le touriste soit conscient avant de s'embarquer sur le traversier de ce qu'il va trouver sur l'île. Gina Vézina met l'accent sur la nature, «à quelques personnes près, qui vont trouver ça plate parce qu'ils disent : "Mais qu'est-ce qu'il y a à faire ici ?" Bien on est sur une île, on est 
entouré d'eau, il y a full nature. Si vous n'avez pas pensé que c'était les oiseaux, la tranquillité, les couchers de soleil qui sont exceptionnels, alors [les touristes] sont pas à la bonne place à ce moment-là ». Dans son cadre insulaire, l'île ne suffit pas toujours à combler les attentes. Car, plutôt qu'une série d'attraits, peut-être s'agit-il avant tout d'un état d'esprit, l'insularité ? L'environnement, le repos, la différence, ce sont des éléments qui ne s'apprivoisent pas en une journée. Dans la mise en place d'un tourisme culturel, que faut-il considérer? L'activité ou le touriste, c'est-à-dire un individu dans un état de réception à l'Autre :

Que tu sois une journée ou que tu prennes un gîte ou une auberge, ou que tu fasses du camping, y faut que tu apprennes à vivre au gré des marées. Alors, tu n'es pas pris sur l'île. Tu n'es jamais pris, tu peux partir dans cinq minutes comme ça si par hasard y faut que tu partes. Tu as juste à prendre l'avion. L'avion, il va être là dans cinq minutes pour te chercher. Mais c'est coûreux, tandis que la traversée est gratuite. Bon ça, avec des sous, tu vas où tu veux, tu vas partout. Alors, tu n'es pas pris, tu n'es absolument pas pris. Sauf qu'il faut que tu apprennes à vivre au gré des marées... Par exemple, les gens qui s'en viennent en vacances une semaine ou deux, qui ont loué un chalet ou quelque part, si vous tolérez ça, vous allez faire des belles vacances. Si vous ne stressez pas parce que vous avez envie d'aller l'autre côté puis le bateau est juste à deux heures de l'après-midi, tu ne peux pas chialer du matin jusqu'à deux heures de l'après-midi parce que le bateau part à deux heures. Tu sais, une fois que tu as compris f̧a puis que tu fais autre chose en attendant, tu n'es pas supposé avoir de problème. C'est la vie des insulaires. Moi, je ne trouve pas que c'est un bon mot quand ils nous disent qu'on est isolé, parce que tu peux le voir de toute sorte de façon l'isolation. On n'est pas en quarantaine icitte. Tu sais on n'est pas en quarantaine, tu es isolé d'une certaine façon, mais y suffit de ne pas paniquer pour l'heure que tu veux sortir de l'île (Gina Vézina).

En tout état de cause, "c'est le prix qu'ils ont à payer pour profiter d'un endroit dont l'isolement apporte un charme particulier" (Vézina 1994 : 131). Cependant, avec les demandes des touristes et leur affluence, c'est toute l'authenticité de l'île qui est mise en jeu. Mais au premier lieu des attentes, c'est la traverse qui cause le véritable choc.

\section{Le tourisme insulaire et l'accessibilité}

Première rencontre avec l'île aux Grues, l'accès maritime constitue à la fois un symbole de coupure avec le continent et une expérience. 
Cette première aventure contient toutefois un revers, donnant lieu à des motifs de frustration. En fait, il apparait que le principal problème du tourisme à l'île est lié au transport. Certes, il y a un service de traversier public gratuit du printemps à l'automne, offrant des départs journaliers. Or, s'il ne doit débourser de sous, le touriste doit se conformer à l'horaire de la traversée. En effet, la traverse est assujettie aux marées et le nombre de places disponibles à bord du traversier est limité. "S'ils ont trop attendu ou s'ils ont manqué le bateau ou si, par hasard, ils n'ont pas été capables d'embarquer avec leur auto sur le bateau, ça crée des frustrations" (Gina Vézina). Voilà bien un problème typiquement insulaire, car l'homme n'a pas d'incidence sur les marées et le touriste doit demeurer un temps précis, mais pas autodéterminé, sur l'île. Le touriste est captif, la durée de la visite étant imposée par le traversier.

Parce que des fois, les gens y se ramassent, ils sont pris un peu sur l'île, soit, ils n'ont pas beaucoup de temps, ou soit ils ont beaucoup beaucoup beaucoup de temps. L'île aux Grues, ce n'est pas un endroit où tu vas passer nécessairement dix heures dans une journée, dans le sens que, une fois que tu as fait le tour, que tu as visité les petits musées, tu es allé à la fromagerie, si tu ne couches pas là puis que tu n'as pas un repas là, bien les gens souvent, un trois à quatre heures, ils en ont assez. Mais le traversier lui, il s'en fout lui du trois à quatre heures. En fait, il s'en fout complètement parce que lui, il a pas le choix : c'est la marée qui lui dicte ses départs. Cela fait que des fois, il y a ça, les gens trouvent ça difficile (Amélie Ringuet).

Pour le touriste le retour peut s'avérer un problème majeur. Pour ceux qui traversent avec leur voiture, la difficulté s'accentue d'un certain niveau de stress. En effet, les places étant limitées à vingt-quatre véhicules sur le traversier, la problématique de l'horaire des marées se conjugue à celle de l'impossibilité de garantir un retour le même jour : "Il y avait beaucoup de touristes, il fallait s'en aller très très de bonne heure au quai si tu voulais ravoir ta place. Si tu ne voulais pas coucher là " (Bernadette Leblond). Car après tout, il n'y a pas que les touristes qui voyagent sur la traverse, les insulaires aussi retournent parfois vers Montmagny avec leur véhicule.

Puis aussi, comme les gens de l'île veulent sortir, bien c'est sûr qu'eux autres, ils stationnent leurs voitures de bonne heure, puis le touriste lui, quand il est en voiture, bien il ne stationne pas nécessairement son auto là deux heures avant le départ du traversier. Surtout s'il veut prendre le temps pour visiter... Mais ça c'est notre travail à nous ici au bureau d'information de bien leur expliquer ça et de leur conseiller 
de ne pas traverser en voiture à la limite, de traverser à pied ou de traverser en vélo, parce que là, ça va être plus facile. C'est qu'au niveau du transport, c'est complexe (Amélie Ringuet).

Encore une fois, l'appréciation du séjour est tributaire de l'état d'esprit dans lequel le touriste appréhende l'île: "Ça dépend de quelle manière le touriste va sur l'île. Je te dirais que ceux qui y vont l'été pour passer une journée complète puis qu'ils ne traversent pas avec leurs vélos, souvent, ils ont hâte de ressortir, fait qu'ils sortent en avion ... ça arrive souvent que l'on va rechercher des touristes à l'île, puis ils disent: "Ben là, on n'avait plus rien à faire là, on ressort." Ce commentaire-là, y revient " (Jean Gosselin). Il est possible de concevoir le désœuvrement des touristes, emprisonnés comme des insulaires, mais peut-être est-ce le seul partage du mode de vie des gens de l'île qu'ils pourront expérimenter dans cette expérience touristique?

\section{Le tourisme culturel et l'authenticité}

«Un attrait qui réussit, un lieu qui est plein de touristes devient nécessairement un peu corrompu et n'offre plus suffisamment de garanties d'authenticité »(Arcand 1993: 75). Or, que soutiennent les promoteurs sinon la perpétuation d'une authenticité toute gruoise? Comment réagir à ces propos publiés dans la carte touristique Excursion à Isle-aux-Grues. Entre temps et marées (2006) qui soutient à des fins publicitaires que "l'Isle-aux-Grues est un véritable havre de paix qui dissimule toutes les traditions et les savoir-faire d'antan», que "[les] insulaires ont su conserver tous les trésors de ce passé rythmé par les marées estivales et les glaces hivernales du majestueux fleuve ", en plus de ce petit texte qui va même jusqu'à offrir aux touristes le stéréotype attendu "[d'un] mode de vie resté inchangé depuis des générations " ? Bernard Arcand précise qu' " il n'est jamais facile d'être un objet de curiosité touristique ni de se faire regarder par tous ces étrangers. Il y a d'abord, bien sûr, le dérangement de la quiétude domestique par une bande d'inconnus malpolis qui demandent à photographier vos enfants ou à acheter vos outils de cuisine ou de jardinage. Mais il y a aussi le fait que ces gens ont de vous une idée aussi précise que parfaitement arrêtée »(1993: 79). La cohabitation devient ainsi risquée, un piège accentué par l'arrivée et l'installation d'insulaires d'adoption durant l'été. Les étrangers insularisés viennent changer la donne, car ceux-ci ne véhiculent pas nécessairement la même image de l'authenticité, à la condition que l'on accepte l'idée stéréotypée 
proposée que les insulaires, les «vrais », colportent cette image. Cette grande migration estivale peut devenir une menace de déculturation et donner une image déformée et amplifiée de la réalité. À la vie quotidienne des insulaires est confrontée l'image idéalisée faite par des vacanciers, mais aussi véhiculée par les promoteurs. Apparemment, les publicités et les infrastructures mises en place afin d'attirer le touriste atteignent leurs objectifs.

«Le tourisme, je pense que c'est la manne pour eux autres. Toutes les places qui semblent se vider un petit peu, bien l'été, les Bed \& Breakfast, les restaurants là-bas, il n'y en a pas à tonne, mais ça doit tourner pas à peu près" (Johanne Ouellet). Certes, l'île est populaire. L'on peut cependant se questionner sur la viabilité de la cohabitation entre la promotion faite de trêve, de vie paisible, avec la masse touristique. Les touristes, l'été, "c'était pleine rue" (Bernadette Leblond). De plus, ils s'interrogent sur leur propre présence, et se questionnent sur l'irritation qu'ils peuvent causer aux gens de l'île: "Peut-être que là-dessus, le moment l'été, un moment fort, peut-être bien qu'ils trouvent ça un peu tannant, mais après ça, peut-être qu'ils regrettent »(Bernadette Leblond). Certains visiteurs soulèvent même le paradoxe entre l'envahissement des touristes et la préservation de leur identité insulaire: "C'est comme une microsociété, ils ont gardé les traditions, puis je trouve ça bien bien correct. Mais je me dis juste qu'il ne faut pas que ce soit envahi par le tourisme " (Johanne Ouellet). Les insulaires doivent durant l'été s'adapter à l'arrivée de l'espèce envahissante que sont les touristes, et comme le souligne Bernard Arcand, «heureux le touriste qui a tout vu avant l'arrivée des touristes!» (1993: 75). Or, les promoteurs touristiques de l'île, malgré la conscience de ces dualités, ne peuvent fermer leurs portes au tourisme, car il ne s'agit plus d'une source nouvelle de revenus, mais bien d'un élément désormais implanté dans l'économie actuelle. Reste à soulever la question à savoir si le jeu en vaut la chandelle et s'ils pourront longtemps jouer sur la typicité de l'île et y amener un amas de touristes, car le tourisme culturel se prête mal à une sorte de tourisme de masse (Ramos 1999 : 291). Le développement du tourisme en milieu insulaire " ne porte-t-il pas en lui-même le germe de sa destruction? " (Defert 1988 : 57). La popularité du lieu est tributaire de l'esprit de tranquillité qui y règne, mais alors comment vivre cette expérience entourée de monde qui déferle dans les rues, d'un relatif isolement envahi de touristes? 


\section{Conclusion}

\section{Le caractère unique d'une ressemblance}

"Chaque île est un rêve et un monde à la fois. Un monde rassurant parce qu'on peut en faire le tour, connaître ses bornes. Telle île est l'aune d'une autre. Les îles se mesurent entre elles, voilà pourquoi elles forment des familles d'îles, avec des personnalités différentes et un air de famille » (Croteau 1995 : 7). Le cas de l'île aux Grues est loin d'être unique, d'une histoire singulière ; elle affiche des similitudes avec d'autres îles. Voisine de l'île aux Coudres, on peut y relever certaines ressemblances dans la promotion d'un tourisme culturel qui met en valeur le côté insulaire des lieux. De prime abord, toutes deux font état d'une économie en bonne partie redevable au tourisme. Bien que la mi-carême ne se pratique plus sur l'île aux Coudres, elle a néanmoins fortement marqué l'imaginaire collectif (Perrault 1962; 1963; 1964; 1999). L'Île aux Grues et l'Île aux Coudres ont réinvesti leur goélette échouée, l'une en salle à manger, l'autre en musée ${ }^{26}$. Toutes deux ont eu comme résident leur peintre de renom, Jean-Paul Riopelle à l'île aux Grues et Jean-Paul Lemieux (1904-1990) à l'Île aux Coudres (Pilon 1986). Elles ont leur musée sur les us et coutumes de leur vie marquée par l'insularité et une tour d'observation qui surplombe l'île. Toutes deux ont chevauché l'hiver avec les canots à glace (Bonnière et Perrault 1959) et sont désormais desservies par un service de traversier ${ }^{27}$. Enfin, on y suggère d'en faire le tour à bicyclette, et elles accueillent les touristes dans leurs gîtes, auberges et campings. L'Île aux Grues n'est donc pas seule à promouvoir un tourisme culturel tributaire de sa géographie.

\section{Le devoir de mémoire}

"Le devoir de mémoire est le devoir des descendants, et il a deux aspects: le souvenir et la vigilance. "(Augé 1998: 120). L'île, sans s'être figée dans le temps, a conservé une certaine authenticité en voulant mettre en valeur ses traditions, son unicité, son caractère, pour la population locale, mais aussi pour l'étonnement de l'invité, sans toutefois tabler sur l'image d'une identité recluse, d'un lieu autre renfermant les savoirs perdus. L'île offre sa culture comme produit et le tourisme culturel, qui est un lieu pour se dire et se définir, devient une

26. Le Musée Les Voiture d'eau à l'Île aux Coudres.

27. Sans pour autant avoir les mêmes difficultés issues des marées. 
ressource complémentaire à l'économie locale. L'île tire avantage de son insularité. On ressent son identité maritime par le transport ainsi que l'exposition de la tradition de l'adaptabilité des insulaires à leur milieu avec les canots à glace. La mise en valeur de cette microsociété et de ses habitants est aussi visible par la mi-carême, attrait offert en dehors de la saison touristique. Le touriste peut ainsi acquérir une connaissance de ce territoire au cours de son séjour. "C'est un peu le paradis finalement " (Johanne Ouellet). Mais en contrepartie, elle n'offre que son insularité au consommateur : "C'est un paradis, mais faut que tu sois conscient de dealer avec tout ça” (Gina Vézina). L'île a su conserver son authenticité, au plus grand plaisir du voyageur, mais le touriste doit accepter les inconvénients de l'isolement tant recherché. Et c'est à lui de prévoir ses besoins, comme en avertit la carte touristique Excursion à Isle-aux-Grues. Entre temps et marées (2006) : "Prenez note qu'il n'y a pas de guichet automatique sur l'île, seulement un comptoircaisse, il est donc recommandé de prévoir de l'argent liquide". L'île revendique son insularité, non pas comme devanture publicitaire, mais comme mode de vie. La contemporanéité de l'île reste l'expression d'une culture insulaire au quotidien avec un tourisme intégré à l'économie, assurant ainsi une plus grande possibilité à l'île de demeurer habitée à l'année afin de promouvoir ce que les insulaires ont été, mais aussi ce qu'ils sont devenus. Cependant, dans quelle mesure le tourisme culturel constitue-t-il une limite? Sommes-nous devant un paradoxe où, pour attirer le touriste, les insulaires doivent maintenir une image du passé, tout en continuant à vivre au présent?

Cette réflexion sur le potentiel touristique de l'île aux Grues révèle en filigrane un nouveau questionnement qui mériterait une enquête ultérieure. Le cas du peintre Jean-Paul Riopelle soulève un fait intéressant dans la construction de la promotion touristique à l'île, puisque, outre les œuvres reproduites sur les fromages, le travail de l'artiste n'est pas exposé dans les guides ou les dépliants touristiques. Ce qui prime, c'est l'homme, la dimension de son œuvre picturale semble évacuée du discours touristique. Le Musée Le Grenier de l'Isle présente et documente principalement l'homme et non son travail. Les touristes ne semblent pas charmés par sa peinture qui célébrait dans son choix iconographique une symbiose entre la création et l'Île aux Grues; ce qu'ils souhaitent avant tout, c'est "d'aller voir sur les lieux où il a vécu " (Bernadette Leblond). L'île semble rejeter la dimension poétique de l'œuvre du peintre pour se consacrer sur sa personne. Une analyse du lien que peut entretenir dans son choix iconographique la peinture de Riopelle et la 
promotion touristique de l'île soulève ici l'interrogation du chercheur. Scruté sous le regard ethnologique, tout ce pan de la recherche reste donc à être approfondi. 


\section{Références}

Arcand, Bernard et Serge Bouchard, 1993, «Le tourisme ». Dans Quinze lieux communs : 71-81. Montréal, Les Éditions du Boréal.

Association touristique régionale de Charlevoix et Tourisme Québec, 2005, Charlewoix. Guide touristique officiel 2005-2006.

Augé, Marc, 1998, Les formes de l'oubli. Paris, Éditions Payot \& Rivages. Béchard, Auguste, 1902, Histoire de l'île-aux-Grues et des îles voisines. Arthabaska, Imprimerie de La Bataille.

Bernier, Robert, 2002, "Jean-Paul Riopelle 1923-2002. Monument international ». Voir semaine du 21 au 27 mars : 12.

Billy, Hélène de, 1996, Riopelle. Montréal, Éditions Art Global.

Bornais, Marie-France, 2003a, "Le Riopelle-de-l'Isle. Inviter une star à sa table ». Le Journal de Québec. Dimanche 28 décembre: 42.

_, 2003b, "Un paradis méconnu ». Le Journal de Québec dimanche 28 décembre: 42 .

- 2003c, "Un terroir unique ", Le Journal de Québec dimanche 28 décembre: 42 .

Bouchard, Claude et Benoît Lacroix [texte de présentation], 2004, Par monts et par vaux: Chaudière-Appalaches. Québec, Les Publications du Québec.

Carignan, Gille, 2002, "C'était quelqu'un comme nous" Si le monde des arts a perdu un géant, les résidants de l'Isle-aux-Grues ont perdu un voisin et un chum ". Le Soleil. Cahier spécial jeudi 14 mars : Z3. Centre local de développement (CLD) de la MRC de L'Islet et l'Office du tourisme de la Côte-du-Sud (MRC de Montmagny), 2006, La Côte-du-Sud. Histoire de vivre. Guide de séjour.

Comeau, Marie-Hélène, archives, "L'héritage des créateurs du Refus Global ", L'aurore boréal, www.afy.ca/aurore/Archives.htm (consulté en avril 2006).

Commission de toponymie du Québec, 2006, Noms et lieux du Québec : dictionnaire illustré. Québec, Les Publications du Québec.

Corporation de développement touristique de L'Isle-aux-Grues, www.isle-aux-grues.com (consulté en avril 2006).

Croteau, André, 1995, Les îles du Saint-Laurent. Saint-Laurent. Éditions du Trécarré.

Defert, Pierre, 1988, Problématique du tourisme insulaire. Cahiers du tourisme. C $58: 1-58$.

Deschênes, Gaston, 1991, La Côte-du-Sud, cette inconnue. Québec, Éditions du Septentrion.

-, 2001, Les voyageurs d'autrefois sur la Côte-du-Sud. Québec, Éditions du Septentrion. 
Dubé, Catherine, 2000, "Des îles et des mythes ». Continuité $85: 25$ 28.

Du Berger, Jean, 2001, "D'hiver en printemps ». Cap-aux-Diamants $64: 9$.

Excursion à Isle-aux-Grues. Entre temps et marées, Carte touristique, 2006. Tourisme Chaudière-Appalaches et Tourisme Québec, 2006, ChaudièreAppalaches. Guide touristique officiel 2006-2007.

Fernandez, Annie, 2002a, « Des amis accompagnent le géant jusqu'à la fin ». Le Journal de Québec jeudi 14 mars: 3.

----, 2002b, «Il voulait mourir dans son manoir ». Le Journal de Québec jeudi 14 mars: 2.

— 2002c, «Un fromage en son honneur». Le Journal de Québec jeudi 14 mars: 2 .

Gauthier, Serge, 2003, "Au sujet du film Père et fils. Folklorique Charlevoix?". Le Devoir vendredi 3 octobre: A9.

Genest, Bernard et Camille Lapointe, 2004, Le patrimoine culturel immatériel. Un capital social et économique. Québec, Ministère de la Culture et des Communications.

Landry, Bérangère, 2001, "Vous prenez t'y des mi-carêmes? ». Capaux-Diamants. 64 : 20-25.

Laviolette, Karine, 2004, "Tourisme culturel et milieu minoritaire:

Un voyage chez les Fransaskois". Ethnologies 26 (2): 259-273.

Lavoie, Richard, 2001, “Du transport au sport. Le canot à glace ». Cap-aux-Diamants. 64 hiver : 29-34.

Le Menestrel, Sara, 1999, "Le tourisme francophone en Louisiane: Un enjeu identitaire". Ethnologies 21 (1): 133-161.

--, 2002, "L'expérience louisianaise. Figure touristique et fauxsemblant ". Ethnologie française 32 (3) : 461-473.

Lemieux, Jean-Marie, 1978, L'île aux Grues et l'île aux Oies. Les îles, les seigneurs, les habitants, les sites et monuments historiques. Montréal, Éditions Leméac.

O’Neil, Jean, 1991, L'île aux Grues. Montréal, Éditions Libre Expression. Pelletier, Claire, 2003, «Le fleuve aux souvenirs ». L'Actualité 28 (11) 94-95.

Perrault, Pierre, 1964, "La mi-carême à l'Ile-aux-Coudres ". Le magazine Maclean 4 (3): 25-28, 30 et suivantes. Archives de folklore et d'ethnologie de l'Université Laval, Fonds Pierre Perrault, P319/D2, 6.

_ 1996, Cinéaste de la parole : entretiens avec Paul Warren. Montréal, Éditions de l'Hexagone. 
— 1999, Nous autres icitte à l'île. Montréal. Éditions de l'Hexagone. Picard, Michel, 1992, Bali. Tourisme Culturel et culture touristique. Paris, L'Harmattan.

Ramos, Francisco Martins, 1999, "Du tourisme culturel au Portugal ». Ethnologie française 29 (2) : 285-293.

Rémillard, François et Benoit Prieur [Portrait], 2003, Le Québec. $8^{\mathrm{e}}$ édition, Montréal, Guides de voyage Ulysse inc.

Réseau des services d'archives du Québec. Réseau de diffusion des archives du Québec; Coutumes et culture, mars, "Carême et micarême", www.rdaq.qc.ca (consulté en avril 2006).

Richard, Jos.-Arthur, 1971, Histoire de Cap St-Ignace. 1672-1970. CapSt-Ignace, La Fabrique de Cap-St-Ignace.

Rimbaud, Arthur, 1992, "Le bateau ivre». Dans Poésies. Une saison en enfer. Illuminations: 68-71. Paris, Éditions J'ai lu.

Ruel, Sylvie, 1996, "L'île masquée s'amuse ». L'Actualité 21 (9) : 42. 45.

1997, "L'archipel de l'île aux Grues ». Le Bel âge 10 (7) : 115 120.

_ 2002 , «Les îles idylliques du Bas-Saint-Laurent ». Le Bel âge 16 (1) : 136-142.

, 2005, "Venez voir les mi-carêmes!". Touring LXXXIII (1): $10-11$.

-----, 2006, "Chaudière-Appalaches. Au fil du fleuve». Touring LXXXIV (1) : 10-14.

Saint-Pierre, Jacques, 2000, La Côte-du-Sud. Québec, Les Presses de l'Université Laval et IQRC.

Simier, Paul, 2005, "La Côte-Nord. La réalité de La grande séduction ", Le Journal de Québec samedi 23 juillet: 29A.

Stanton, Julie, 1999, «L'Isle-aux-Grues, un paradis!». Le Bel âge 12 (6) : 104-110.

Tourisme Chaudière-Appalaches, www.chaudiereappalaches.com (consulté en avril 2006).

Tourisme Isle-aux-Coudres SETIAC, www.charlevoix.qc.ca/isle-auxcoudres (consulté en avril 2006).

Tremblay, Régis, 2002, "Dernier envol d'un rebelle. Jean-Paul Riopelle s'éteint à l'Isle-aux-Grues à l'âge de 78 ans". Le Soleil jeudi 14 mars : A1-A2. 
Turgeon, Laurier, 2003, "Le paysage. Construire une "ethnoscopie" Basque au Québec". Dans Laurier Turgeon (dir.), Patrimoines métissés. Contextes coloniaux et postcoloniaux: 129-160. Québec et Paris, Les Presses de l'Université Laval et Éditions de la Maison des sciences de l'homme.

Vézina, Jules, 1994, La Côte-du-Sud. Histoire et généalogie d'un archipel.

Île aux Grues, Association historique.

Viau, René, 2002, "Un hibou dans la nuit », Le Soleil, cahier spécial, jeudi 14 mars : Z1-Z2.

Sources audiovisuelles

Bonnière, René et Pierre Perrault, 1959, La Traverse d'hiver à l'île aux Coudres. Crawley Films Ltd. pour Radio-Canada, Coll. "Au pays de Neufve-France".

Caron, Myriam, 2005, "Le sacro-saint tourisme». Dans Jean-Évrard Bilodeau, Janie G. Brunet et Catherine Genest (dir.), Méchant contraste. Télé-Québec.

Feaver, Marianne et Pierre Letarte, 1982, Riopelle. Office national du film.

Garceau, Raymond, 1958, Une Ile du Saint-Laurent. Office national du film.

- 1965, Îles-de-la-Madeleine: tourisme et transport. Office national du film.

Gaston, Claire et Louis Lalande, 1994, "Riopelle ». Le Point. Société Radio-Canada.

Laliberté, Éli et Patrick Guité, 2006, Mi-Carême. Mimaji production. Lavoie, Richard, 1963, Noël à l'île aux Grues. Les Films Lavoie Inc. pour Radio-Canada.

$\longrightarrow, 2003$, Le temps des madelinots. Richard Lavoie inc. et l'Office national du film.

Pelletier, Jacques, 1994, Prenez-vous des Mi-Carêmes? La fête de la micarême à l'Ile-aux-Grues. Centre de valorisation du patrimoine vivant, Carrefour mondial de l'Accordéon et Municipalité de l'Ile-auxGrues.

Perrault, Pierre, 1962, La mi-carême à l'Ile-aux-Coudres. Archives de folklore et d'ethnologie de l'Université Laval, Fonds Pierre Perrault, P319/D2, 6, SPV.326.

— - et Michel Brault, 1963, Pour la suite du monde. Office national du film. 
Pilon, Huguette, 1986, "L'univers de Jean-Paul Lemieux». Le Point. Société Radio-Canada.

Pouliot, Jean-François, 2004, La grande séduction. Max Films inc. 